\title{
IMPROVED WI-FI SIGNAL QUALITY INDICATOR USING WI-FI PERFORMANCE OBSERVATION
}

\author{
Ahmad Tajuddin Samsudin a , Mohd Shahril Saharedan b, Syaiful Nizam Yahya c, \\ Mohd Syafarees Ishak ${ }^{d}$, Siti Salwa Said e \\ abcde Telekom Research \& Development, Cyberjaya, Malaysia \\ Corresponding email: tajuddin@tmrnd.com.my
}

\begin{abstract}
Nowadays, the customer uses a device that requires wireless connectivity either outside or inside the house. The customer usually relies on the device indicator that refers to the received signal strength indicator (RSSI). On the device, the RSSI is designated as bars rating the Access Point (AP) signal strength for the customer. Most customers of Wi- Fi devices will check the AP signal quality indicator before they connect to the AP. However, the RSSI can be confusing to the customer. This is because customers often associate the Wi-Fi performance with RSSI, with the perception that the higher RSSI, the better performance of data transmission. This perception somewhat true, however, sometimes that perception can be denied by interference, such as electromagnetic signal, congested frequency by others AP, and congested traffic by other devices connecting to same AP. Therefore, this paper proposed a new indicator to provide a real guidance to the customer, which introduce signal quality indicator which combine actual RSSI and Wi- Fi performance, such as latency and loss to represent the actual performance of the AP. Based on the observed data latency, loss and RSSI, we perform a regression analysis for curve fitting. The result shows that the proposed signal quality indicator is better than the actual RSSI rating bar to reflect the Internet experience.
\end{abstract}

Keywords: Signal Quality Indicator; Wi-Fi Performance; Regression Analysis; RSSI \& Access Point.

\section{Introduction}

Nowadays, the customer uses a device that requires wireless connectivity either outside or inside the house. The customer usually relies on the device indicator that refers to the RSSI. On this device, the RSSI is designated as bars rating the AP signal strength for the customer. Most customers of WiFi devices will check the AP signal quality indicator (Figure 1) before they connect to the AP. The majority of customers will tend to choose a full bar [Figure 1(a)] to make an Internet Connection while most will try to avoid Figure 1(c) and 1(d) while making connections to the AP.

It is human nature to choose the strongest AP signal quality, whether it has the characteristics of the securities or not. Sometimes, if the indicator shows the bar as Figure 1(c) and 1(d), the customer has no option but to choose that AP and then try to get as close as possible to it so that the signal becomes as strong as Figure 1(a) or at least 1(b).

If you are an iPhone customer, maybe you still remember the iPhone 4 issues regarding the signal quality indicator drop-in bar. According to Apple press, the algorithm for calculating the signal strength was inaccurate, showing more bars than it should have shown in some cases (Apple Inc, 2010). In an example provided by Apple, the signal strength displayed on your iPhone 4 could be two bars higher than the actual signal strength. Apple's statement also 
suggests that the real signal strength never changes. Thus, when customers see the low signal strength from placing pressure along one of the antennas, they are really seeing a more accurate representation of the signal.

Generally, a customer may know that signal strength is influenced by two main factors: Distance and physical object. The farther the Wi-Fi device is from the AP, the lower the RSSI. Our findings are based on 10 different RG models with a line of sight that showed the RSSI will be lower than $-55 \mathrm{dBm}$ when reaching a distance of 50 to 90 meters (Tajuddin, 2017). Obstructions like walls, (e.g. stone, concrete or brick) glass, metal, or water may also reduce the quality of radio signals between the $\mathrm{AP}$ and Wi-Fi devices. These considerations can result in the customer experiencing slow Internet service and intermittent connection problems. Obviously, in the above situation, the customer will have to know how to get a full bar signal if faced with problems of distance and physical object interference.

Few customers realize that in certain circumstances, although the indicator shows a full bar [Figure 1(a)], they may still have difficulty getting the best Internet experience. This excellent signal strength can be blocked by interferences such as electromagnetic signals, congested frequency by other APs, and congested traffic by other devices connecting to the same AP.

There are two types of interference: AP and non-AP. AP interference which consists of three types of interferences: Co-channel, adjacent-channel and self-interference. AP interference occurs when multiple access points are nearby and the channels used by the access points overlap. Co-channel interference comes from other APs that use the same channel to communicate. Adjacent-channel interference comes from APs that use different channels. Selfinterference occurs when one or more client devices are connected into AP or AP repeater.

If you are the AP owner, you can manage those devices by either connecting to your AP using the RG's admin web page or limiting the number of the devices. However, if you access the AP belonging to your company or one that is public, you cannot manage the AP. You need to compete with other customers to get bandwidth. If the AP's admin allows all ports, then you will face issues on wireless performance, and thus rely on the behavior of the customer using that AP. For example, just browsing simple web pages and working with email consumes only a small portion of bandwidth, even if ten customers are doing so at the same time. However, streaming video from YouTube or heavy downloads of torrent files may dramatically degrade Internet experience.

A second category of interference comes from electromagnetic signals from appliances such as microwave ovens, wireless baby monitors, cordless phones, alarm systems, etc. These appliances mainly use the $2.4 \mathrm{GHz}$ frequency of channels 1 to 13 (F. Kaabi et al., 2010). Electromagnetic signal interference does not degrade the RSSI, but it will degrade the data transmission performance (Li Yingxiong et al., 2016). The symptom can be observed via wireless measurable performance metrics such as loss, latency and jitters. The customer will feel the impact of slow Internet experience and intermittent connection problems.

In this paper, we will (1) discuss the related RSSI and wireless performance indicator used by the operating system, (2) propose a new signal quality indicator, (3) set up a simple experiment to show the comparison between the existing and a new indicator, and (4) end with a discussion and conclusion. 


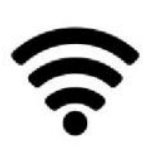

(a)

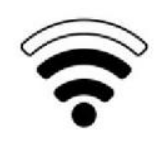

(b)

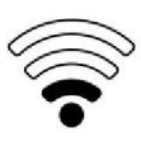

(c)

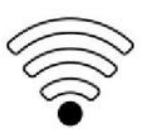

(d)

Figure 1: Wi-Fi signal quality indicator of access point

\section{Related works}

Has your phone's signal quality indicator ever tricked you into thinking you have better reception than you actually do? Usually, the signal strength is represented as a quality in percentage (o to 100\%), an RSSI value in $\mathrm{dBm}$, or a rating bar, depending on your operating system and the application which you use.

If you are a Windows customer, the operating system provides you with a wireless rating bar on the Windows taskbar. This lets you say something about the signal strength based on the bar. Microsoft Developer Resources uses percentage to represent the signal quality bar(WLAN_Available Network Structure, 2016). The percentage contains a value between o and 100. A value of o implies an actual RSSI signal strength of $-100 d B m$. A value of 100 implies an actual RSSI signal strength of $-50 \mathrm{dBm}$. If we compare the RSSI value with the rating bar, we can say that anything better than or equal to $-50 \mathrm{dBm}$ will show the full bars and anything worse than or equal to $-100 \mathrm{dBm}$ will show o bars.

The Android developer community provides a public API called the WiFi Manager Class. This class provides the primary API for managing all aspects of Wi-Fi connectivity. The android developer can use this public API to compute the signal quality level. However, the full bar in an android is different from the Microsoft bar. Here, anything better than or equal to $-55 \mathrm{dBm}$ will show the max bars, whereas anything worse than or equal to -10odBm will show o bars. To draw a wireless bar for signal quality, we can use the static method shown below:

\section{Wi-Fi Manager. Calculate Signal Level (RSSI,x);}

where $\mathrm{x}$ is the signal level. This method returns a number between $\mathrm{o}$ and ( $\mathrm{x}-1)$. For example, if you want to draw 4 bars, then you should set $x=4$.

In summary, Figure 2.0 shows how an operating system and application display the Wi-Fi signal quality indicator. 


\begin{tabular}{|c|c|c|c|c|c|c|}
\hline Windows 10 & \multicolumn{2}{|c|}{ iPhone 5s } & \multicolumn{2}{|l|}{ Android 4.4} & \multicolumn{2}{|c|}{ CentOS 7.2} \\
\hline ofe tmmand vpn & Wi-Fi & $\mathrm{O}$ & Wifi & $\bullet$ & ged wi-fit & 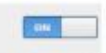 \\
\hline & $\checkmark$ TMRND & $\therefore=$ (1) & consecteo & & 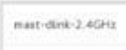 & $0=9$ \\
\hline $\begin{array}{l}\text { Th. mast-dilink-2.4GHz } \\
\text { Connected, secured }\end{array}$ & CHOOSE ANETWORK... & & $\begin{array}{l}\text { TMRND } \\
\text { Tipp to o hare paseavord }\end{array}$ & $\therefore$ & 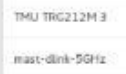 & $\ddot{0}$ \\
\hline 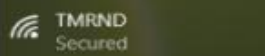 & 1TM_staff & $\therefore=$ (i) & 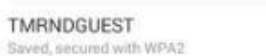 & $\cdot 78$ & тенек & 0.4 \\
\hline $\begin{array}{l}\text { If. } \\
\text { Securstaft }\end{array}$ & itdwlan & $\therefore=$ (i) & AVALLALENETWORKS & & intom & $\begin{array}{l}0.9 \\
0.0 \%\end{array}$ \\
\hline & LEILA & $\therefore=$ (1) & 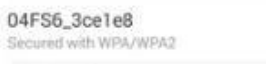 & $\cdot 9$ & & \\
\hline 116. Secured & mast-dlink-2.4GHz & $\therefore=$ (1) & $\begin{array}{l}\text { 1TM_staff } \\
\text { Secured whth wenz }\end{array}$ & $\leftrightarrow 2$ & & \\
\hline ffr. Sinar2 & mast-dlink-5GHz & $\therefore=$ (i) & $\begin{array}{l}\text { dos_santos } \\
\text { Soceured whth wh }\end{array}$ & .96 & & \\
\hline Tf. TMU TRG212M 3 & SSID2-209 & $\theta=$ (i) & g-corn-1@unifi & & & \\
\hline Network settings & TMRNDGUEST & $\therefore=$ (i) & & & & \\
\hline
\end{tabular}

Figure 2: Wi-Fi signal quality indicator on several platforms

Normally, after establishing the connection between the AP and the device, the operating system will display the connection or link speed. However, the customer must perform multiple steps or install a widget to get that information. Figure 3.0 shows several snapshots for various platforms that display link speeds as well as some attributes for Wi-Fi connection.

\begin{tabular}{|c|c|c|c|}
\hline Windows 10 & iPhone 5s & Android 4.4 & CentOS 7.2 \\
\hline 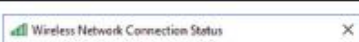 & \multirow{12}{*}{$\begin{array}{l}\text { Airport Utility - } \\
\text { information not available }\end{array}$} & 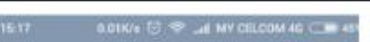 & \multirow{12}{*}{ 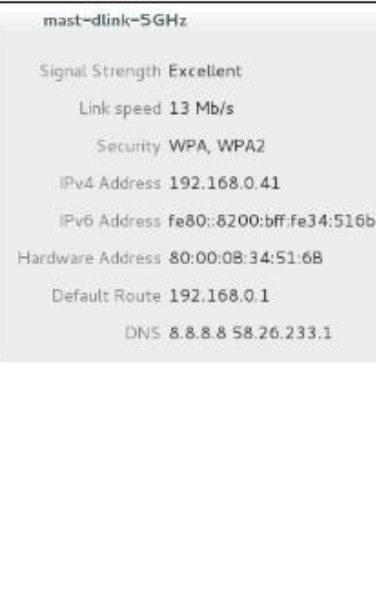 } \\
\hline & & 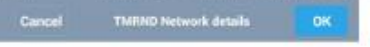 & \\
\hline 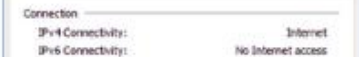 & & Auto connect & \\
\hline 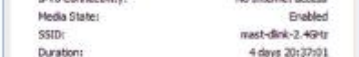 & & Mobile hotspots & \\
\hline nelver & & connected & \\
\hline 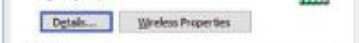 & & & \\
\hline 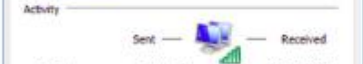 & & Signal strength & \\
\hline 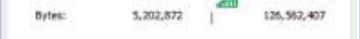 & & Link speed & \\
\hline 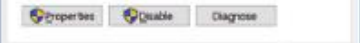 & & WPA2 PSK & \\
\hline \multirow[t]{3}{*}{ ane } & & IP address & \\
\hline & & Subnet mask & \\
\hline & & 10044251 & \\
\hline
\end{tabular}

Figure 3: Snapshot of Link Speed of Wi-Fi on several platforms

The signal quality indicator shown on the taskbar or setting refers only to the actual RSSI. To get the current Wi-Fi performance, the customer must drill down into the network setting or properties to find the Wi-Fi performance. Another alternative for the customer is to use other external applications such as speed test, ping, trace route, iperf, or another network forensic tool(iPerf, 2016). Nevertheless, a speed test [Figure 4(a)] will show the performance between the device and the server in the Internet(Speedtest, 2016). If the server is outside the range of Customer Premises Equipment (CPE), it will not reflect Wi-Fi performance. If a customer 
subscribes to 20Mbps speed, the speed test will give a result 20Mbps or less. However, it is known that the link speed between the device and the access point depends on the $802.11 \mathrm{a} / \mathrm{b} / \mathrm{g} / \mathrm{n} / \mathrm{ac}$ type radio. Besides a speed test, you can monitor Wi-Fi performance by pinging the IP address of the RG. Figure 4(b) shows how to ping from a customer laptop (192.168.0.41) to the RG with IP address 192.168.0.1 as much as ten times. The result shows the packet loss and round-trip time (RTT). On average, the device took $8.424 \mathrm{~ms}$ to send 64 bytes of data from the device to the access point. However, the ping tool does not show the data throughput. These two alternatives are typically done by the ordinary customer.

There are a few studies created to measure the performance of Wi-Fi traffic between the AP and the device, the objective being a best channel assignment and path computation (for best link or routing)(Saleem Iqbal et al, 2015). All studies agreed that the most effective measurement uses performance metrics such as throughput, latency and loss. Because of the highly dynamic nature of Wi-Fi traffic congestion, traffic flow measurement is therefore most effective when performed frequently. Because customers must perform these measurements periodically, they may be inconveniencing. It would be better if the Wi-Fi signal quality indicator was capable of showing performance of AP Wi-Fi every second. It would also be better if the indicator could be displayed in a number of devices connected to that AP.

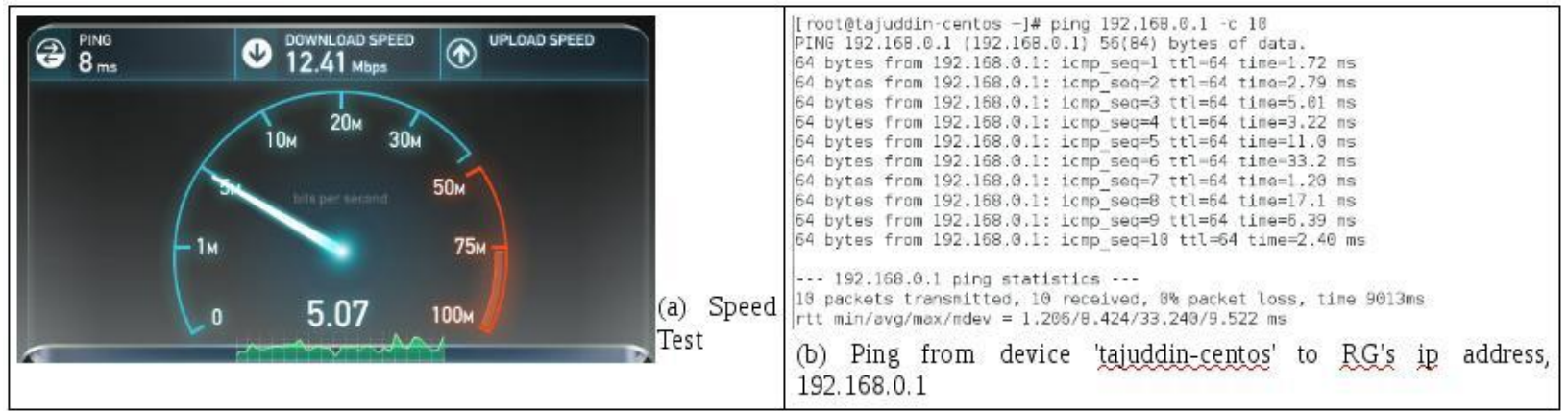

Figure 4: Example network performance tool such as Speed Test and Ping tool

\section{Predicted RSSI}

In this paper, we hypothesize that a correlation exists between the RSSI and Wi-Fi performance (latency and loss). Thus, there are two hypotheses: (i) Hypothesis null 1 (H1) - there is no correlation between the RSSI and latency; (ii) Hypothesis null 2 (H2) - there is no correlation between the RSSI and packet loss.

To get a correlation between the RSSI and latency/loss, we have developed a simple methodology to collect data for RSSI, latency and loss. Therefore, we have developed an experimental setup to the access point (using the RG model - Innatech RG4332) and device (x86 hardware with Wi-Fi 802.11n). This experiment was done in a controlled manner and with no interference from other APs or electromagnetic signals. We used Wi-Spy DBx [17] to detect the presence of noise (electromagnetic signals).

The RSSI observation stems from $-30 \mathrm{dBm}$ to $-80 \mathrm{dBm}$, with an incremental of $-5 \mathrm{dBm}$. Observation of the Wi-Fi performance metric was based on RTT (in milliseconds) and loss (in percentage). To obtain the latency and loss, we used a simple ping command from the device to the AP. We executed the ping command as follows: 
ping 192.168.o.1 -c 30 -s 1500

where $\mathrm{c}$ is the number of packets created and $\mathrm{s}$ is the packet size.

The ping command above was executed 50 times for each RSSI; therefore, we created 1500 icmp packets sized at 1500 bytes per packet. In the end, we had at least a 1500 value of RTT (this can be referred as latency) and a 50 value of loss. From these raw data, we plotted the curve estimation under regression analysis using SPSS. We did two regression analyses: (1) the first set RSSI as an independent variable and latency as a dependent variable, (2) the second set RSSI as an independent variable and loss as a dependent variable. Figure 5.0 shows the estimated curve between RSSI and latency. Figure 6.0 shows the estimated curve between RSSI and loss.

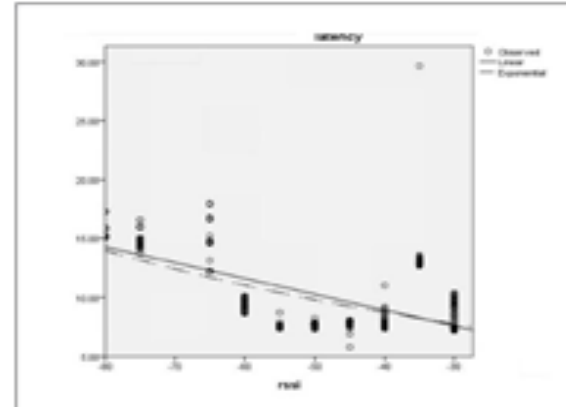

(a) Estimation curve
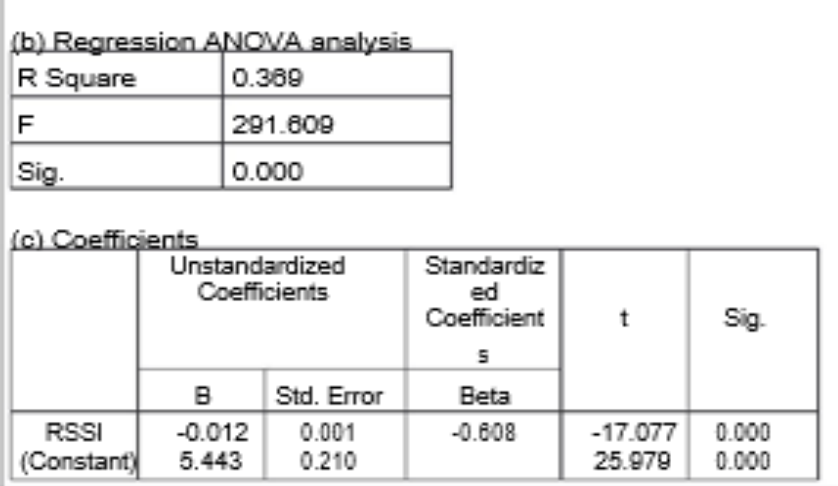

Figure 5: Regression analysis between RSSI and latency

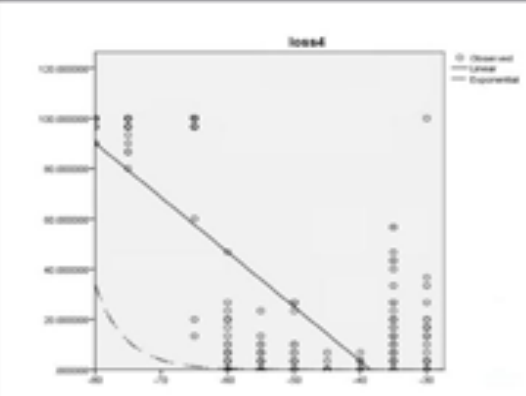

(a) Estimation curve

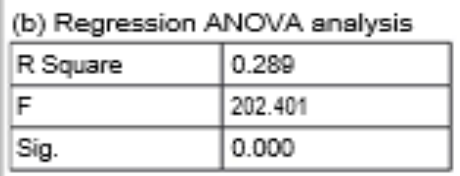

(c) Coefficients

\begin{tabular}{|c|c|c|c|c|c|}
\hline \multirow{2}{*}{} & \multicolumn{2}{|c|}{$\begin{array}{c}\text { Unstandardized } \\
\text { Coefficients }\end{array}$} & $\begin{array}{c}\text { Standardized } \\
\text { Coefficients }\end{array}$ & \multirow{2}{*}{$\mathrm{t}$} & \multirow{2}{*}{ Sig. } \\
\cline { 2 - 4 } & $\mathrm{B}$ & Std. Error & Beta & & \\
\hline RSSI & -0.218 & 0.015 & -0.538 & -14.227 & 0.000 \\
(Constant) & $9.348 \mathrm{E}-7$ & 0.000 & & 1.172 & 0.242 \\
\hline
\end{tabular}

Figure 6: Regression analysis between RSSI and loss

From Figures 5(a) and 6(a), we plotted the exponential model as Equation (1):

$\mathrm{y}=\mathrm{e}^{\wedge}(\mathrm{x} \cdot \mathrm{RSSI}+\operatorname{Ln}($ Constant $))$

However, because we can predict the $\mathrm{x}$ value (where $\mathrm{x}$ is a prediction of RSSI), we can also find the $\mathrm{x}$ by taking the following steps until reaching Equation (2):

$\operatorname{Ln} y=\operatorname{Ln}\left(e^{\wedge}(x \cdot R S S I+\operatorname{Ln}(\right.$ Constant $\left.))\right)$ 
Ln $\mathrm{y}=\mathrm{x} . \mathrm{RSSI}+\mathrm{Ln}$ (Constant)

$\mathrm{x} . \mathrm{RSSI}=\operatorname{Ln} \mathrm{y}-$ Ln (Constant)

$\mathrm{x}=(\operatorname{Ln}(\mathrm{y})-\operatorname{Ln}($ Constant $)) /$ RSSI

Based on Figure 5(c), we can obtain the estimated curve using Equation (1) for correlation between RSSI and Latency:

$\mathrm{y}=\mathrm{e}^{\wedge}(\mathrm{x} .(-0.012)+\operatorname{Ln}(5.443)) \mathrm{y}=$

$\mathrm{e}^{\wedge}(\mathrm{x} .(-0.012)+1.694)$

Thus, to predict the RSSI based on Equation (2), we arrived at the estimated curve

as follows:

$\mathrm{x}=(\operatorname{Ln}(\mathrm{y})-\operatorname{Ln}(5.443)) /(-0.012)$

$\mathrm{x}=(\operatorname{Ln}(\mathrm{y})-1.694) /(-0.012)$

Based on Figure 6(c), we can obtain the estimated curve using Equation (1) for correlation between RSSI and Loss:

$\mathrm{y}=\mathrm{e}^{\wedge}(\mathrm{x} \cdot(-0.218)+\operatorname{Ln}(9.348 E-7))$

$\mathrm{y}=\mathrm{e}^{\wedge}(\mathrm{x} \cdot(-0.218)-16.1855)$

Again, to predict the RSSI based on Equation (2), we arrived at the estimated

curve as follows:

$\mathrm{x}=(\operatorname{Ln}(\mathrm{y})-\operatorname{Ln}(9.348 E-7)) /(-0.218)$

$\mathrm{x}=(\operatorname{Ln}(\mathrm{y})+16.186) /(-0.218)$

Exponential models (3) and (4) have limitations because the dataset of RSSI observation is from $-30 \mathrm{dBm}$ to $-8 \mathrm{odBm}$, and is specific to the Innatech RG4332 model. Therefore, the $y$ values corresponding to the RSSI, using the regression model in this paper, are valid from -3odBm to 8odBm and are specific to the RG Innatech RG4332. To get a better prediction, we suggest collecting more datasets of loss and latency corresponding to the RSSI from -20dBm to $100 \mathrm{dBm}$ with $-5 \mathrm{dBm}$ incremental, and then testing with several RG models.

The ANOVA regression analysis of Figures 5(b) and 6(b) showed that the Significance value was 0.000 for both regression analyses between RSSI and latency, and RSSI with packet loss. Since the $p$ value was lower than 0.05 , we rejected hypotheses $\mathrm{H}_{1}$ and $\mathrm{H} 2$. We can conclude that there is significant correlation between the RSSI and latency, as well as RSSI and packet loss. 
We can use Equation (1), $\mathrm{y}=\mathrm{e}^{\wedge}$ (x.RSSI + Ln (Constant)) for an RSSI prediction. Thus, if we want the $\mathrm{x}$ value corresponding to latency $(\mathrm{y}=10 \mathrm{~ms})$, using the above model $(3)$, we get $\mathrm{x}=(\mathrm{Ln}$ $10-1.694) /(-0.012)=-50.72 \mathrm{dBm}$. If we want to predict the $\mathrm{x}$ value corresponding to packet loss $(0.01 \%)$, using the model $(4)$, we get $x=(\operatorname{Ln}(0.01)+16.186) /(-0.218)=-53.12 \mathrm{dBm}$.

To make life easier for the customer, the predicted RSSI needs to be converted into a rating bar. It is easier for the customer to see changes in the predicted RSSI using a bar rating instead of value of $\mathrm{dBm}$. Again, Figure 1 shows the Wi-Fi signal quality received by the device. Therefore, we suggest including the predicted RSSI into the Wi-Fi signal quality indicator. We can use RSSI mapping for Windows, where anything better than or equal to -50dBm will show full bars, and anything worse than or equal to $-100 \mathrm{dBm}$ will show o bars. Figure 7 shows the suggested signal quality indicator, which includes the actual RSSI [left side] and predicted RSSI [right side]. From this new indicator, customers can monitor the Wi-Fi performance against the actual signal strength received by the device.

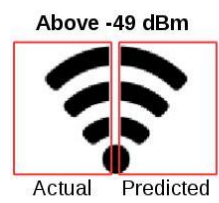

(a)

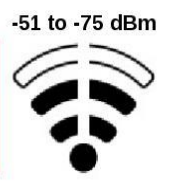

(b)

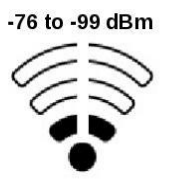

(c)

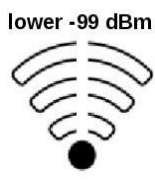

(d)

Figure 7: Newly rating bar for actual and predicted RSSI

\section{Experimental setup}

To prove that the signal quality indicator shown in Figure 1 may mislead Wi-Fi customers, we conducted a simple experiment to show that the full bar of the signal quality indicator [Figure 1(a)] is not necessarily a good indicator of network performance. This experiment was conducted in a controlled space of $10 x 10$ meters. Generally, we wanted to show the differences in Wi-Fi signal quality and performance when using IEEE 802.11n technology where the electromagnetic signal was generated to interfere with the connection established between the device and the AP.

We configured the RG Innatech-RG4332, a laptop and a microwave oven. Table 1 shows the attributes for each device used.

We observed the data in three cases; (i) Condition 1 - microwave oven is OFF; (ii) Condition 2 - microwave oven is ON; and (iii) Condition $3-$ microwave oven is OFF again. 
Table 1: Attributes of devices

\begin{tabular}{|l|l|}
\hline Device & Attributes \\
\hline Residential Gateway & $\begin{array}{l}\text { model: RG-INNATECH-RG4332 } \\
\text { microwave frequency: 2400 to 2484 Mhz } \\
\text { antenna gain: } 3-5 \mathrm{dBi} \\
\text { channel used: } 11\end{array}$ \\
\hline $\begin{array}{l}\text { Laptop - Device } \\
\text { under Test (DUT) }\end{array}$ & $\begin{array}{l}\text { model: HP ProBook } \\
\text { cpu: Intel(R) Core i7-4702MQ CPU @ 2.20GHz x } 8 \\
\text { operating system: centOS 7.2 64 bits } \\
\text { support: 8O2.11 n/ac }\end{array}$ \\
\hline Microwave oven & $\begin{array}{l}\text { model: Nikita MVO-1707 } \\
\text { microwave frequency: } 2450 \mathrm{MHz}\end{array}$ \\
\hline
\end{tabular}

For Condition 1, we configured and enabled the RG to create a Wi-Fi SSID TMU-RG4332, which we set to Channel 11. The device under test (DUT) was placed within 5 meters of the RG without a physical object that could become a barrier between the RG and the DUT. We ensured that no strong electromagnetic signal would interfere with the Channel 11 setting by using Wi-Spy DBx. We installed and mounted the Wi-Spy DBx on the DUT. Similarly, we ensured there was no interference of other APs so as not to disturb the experiment.

After that, we observed the Wi-Fi performance by using the ping command as follows:

ping 192.168.0.1 -c 120 -s 1500

The above ping command produces 120 icmp-sized packets of 1500 bytes each. We executed the ping ten times. The results of this command generated 1200 packets and produced the RTT (or latency) and loss. At the same time, we monitored the RSSI received from the TM-RG4332 SSID. Once we obtained the required reading in Condition 1, we proceeded to Condition 2.

In Condition 2, before turning on the microwave oven, we made sure that there were no other AP or appliance signals. We placed the microwave oven near to the RG, with no obstacle between the RG, DUT and microwave oven. The distance between the RG and microwave oven was $50 \mathrm{~cm}$. There were 5 meters between the RG and the DUT, and between the microwave oven and the DUT.

When we found no other signal interference, we turned on the microwave oven. We set the microwave oven power to high and the timer for 30 minutes. After the microwave oven was on for five minutes, we observed the electromagnetic signal and RSSI by using Wi-Spy DBx and the Wi-Fi performance through the ping command. We repeated the ping command 10 times. 
After completing the observation of Condition 2, we turned off the microwave oven and ensured that any existing electromagnetic signal had been lost. If, after five minutes, we still detected the electromagnetic signal, we replayed the Condition 2 test. We did this is because Wi-Spy DBx cannot distinguish the electromagnetic signal coming from a microwave oven or other sources. Having confirmed that the microwave oven used was the only appliance creating these signals, we proceeded to Condition 3 observations.

During Condition 3 observations, after shutting down the microwave oven and observing no electromagnetic signals after 5 minutes, we continued to observe the Wi-Fi signal quality and performance. The purpose of Condition 3 was to determine whether our observed data were approximately equal to the data observed in Condition 1. During Condition 3 observations, we continued to observe the Wi-Fi signal quality and performance.

Observations from Conditions 1, 2 and 3 gave data on RSSI, latency and loss. We yielded the jitter from latency result and produced the predicted RSSI based on Equations (3) and (4).

\section{Results and discussion}

In Condition 1, 2 and 3's test observations [Table 2(b-d)], we yielded the jitter from latency result and showed that an interference occurred in Condition 2. Here, we saw that the Wi-Fi performance degraded tremendously from Condition 1, but became similar to Conditions 1 and 3 after switching off the microwave oven. Table 2(a) shows that the RSSI in all three conditions is consistent and above $-50 \mathrm{dBm}$. These findings show that while the RSSI received by the laptop was not affected by the electromagnetic signal produced by the microwave oven, this interference signal had a substantial impact on Wi-Fi performance. This was shown by latency increasing from $9.51 \mathrm{~ms}$ to $20.08 \mathrm{~ms}$, packet loss from $0.96 \%$ to $56.64 \%$, and jitter reach to 20.56 $\mathrm{ms}$ from $9.15 \mathrm{~ms}$.

If the customer is in Condition 2, he or she will likely experience slow Internet downloading, browsing, or even more re-buffering during video streaming. When customers check the Wi-Fi signal quality [Table $2(\mathrm{~g})$ ], and the rating is full bar, they might think that the Internet connection is the problem and not the Wi-Fi or the RG. To verify that the Internet connection is the problem, they often use a speed test and check the throughput. Those who are network literate tend to use ping, trace route, or network forensic tools such as Wireshark[18], etc. Some may also try to get wireless information as shown in Figure 3. These extra steps can be avoided if the Wi-Fi signal quality indicator is similar to that shown in Figure 7.

Based on observed Wi-Fi performance, we predicted the RSSI base in Equation (3) and (4). Table 2(e-f) shows the predicted RSSI for all Conditions. From these 
predicted RSSIs, we can draw a proposed signal quality indicator as shown in Table 2(h-i).

Table 2. The results show the comparison output of Condition 1, 2 and 3.

\begin{tabular}{|c|l|c|c|c|}
\hline No & Parameter & Condition 1 & Condition 2 & Condition 3 \\
\hline a & RSSI, dBm & -45.60 & -46.1 & -46.3 \\
\hline b & latency (average). ms & 9.51 & 20.08 & 10.50 \\
\hline c & loss, \% & 0.86 & 56.64 & 0.80 \\
\hline d & iitter, ms & 9.15 & 20.56 & 9.45 \\
\hline e & predicted rssi (based on equation (3)) & -46.52 & -108.81 & -54.78 \\
\hline f & predicted rssi (based on equation (4)) & -74.06 & -92.76 & -73.28 \\
\hline g & signal quality indicator (based on Figure 1) & & & \\
\hline h & new signal quality indicator (based on Table 2(e)) & & & \\
\hline i & new signal quality indicator (based on Table 2(f)) & & & \\
\hline
\end{tabular}

If the device system can show the proposed Wi-Fi signal quality indicator, the customer will know that there is a Wi-Fi performance issue and quickly narrow down the problem. For example, Table 2(i) for Condition test 1 shows that the actual RSSI is high and full bar; however, the Wi-Fi performance is 3 out of 4 bars. When we go to Condition test 2, the customer knows that the Wi-Fi receiver for the laptop and RG antenna works well because the actual RSSI is still high, but the indicator for Wi-Fi performance is one bar. This means that in Condition test 2, there are some issues with Wi-Fi connectivity. While customers don't know the root cause, they will know that they will experience a poor Internet connectivity. They will need to find an answer to why their access point shows poor Wi-Fi performance. The problem may be because of interference signals from other APs or appliances, or other devices sharing the same AP. At least the customer will know that the problem is not in the AP to Internet network.

\section{Conclusions}

The WLAN eco-system is more complicated, with many more APs coexisting either in a centralized or uncoordinated management. We proposed a new Wi-Fi signal quality indicator that considers the actual RSSI and Wi-Fi performance between a device and the AP. The experiment showed that the proposed Wi-Fi signal quality indicator made it easier for customers to compare a normal signal quality indicator during Internet problems due to interference signals from other APs and electrical appliances. Because these interferences will affect Wi-Fi performance but not the actual RSSI, a new rating bar showing actual and predicted RSSI will help customers know if their Wi-Fi has a problem, even though the RSSI on the signal quality indicator shows an excellent reading. Then, if the customer calls the ISP Contact Center for help, the help desk can easily narrow down the problem by troubleshooting the network side beyond the access point.

This proposed indicator could also apply to a scenario where the AP and devices have not established connections between each other. If we could discover how to extend this feature to all other APs, this would be good because customers would be able to choose the best Wi-Fi 
hotspot performance available. Besides that, we want to add the total connected devices into the signal quality indicator so that the Wi-Fi customer knows immediately how many devices are already connected to the same AP. To improve touch point efficiency and effectiveness for the customer, we are currently designing and developing the predicted RSSI based on Equations (3) and (4) on various client platforms such as Android, iOS and Win 10, to be used by the TM customer. Positive customer experience will help retain customers, thus increasing company profits. 


\section{References}

i. Antony Franklin et al, 2012. Online reconfiguration of channel assignment in Multi-Channel MultiRadio wireless mesh networks. Journal of Computer Communication, 35(16), pp. 2004-2013.

ii. $\quad$ Apple Inc, 2010. Letter from Apple Regarding iPhone 4. [Online] Available at: http://www.apple.com/pr/library/2010/07/02Letter-from-Apple-Regarding-iPhone4.html

iii. Apurv Bhartia et. al, 2016. IQ-Hopping: Distributed Oblivious Channel Selection for Wireless Networks. 17th ACM International Symposium on Mobile Ad Hoc Networking and Computing, pp. 81-90.

iv. $\quad$ F. Kaabi et al., 2010. Channel Allocation and Routing in Wireless Mesh Networks: A survey and qualitative comparison between schemes. International Journal of Wireless and Mobile Networks, 2(2), pp. 132-150.

v. InSSider, 2016. InSSider. [Online] Available at: http://www.metageek.com

vi. $\quad$ iPerf, 2016. The ultimate speed test tool for TCP, UDP and SCTP. [Online] Available at: https://iperf.fr/

vii. $\quad$ Li Yingxiong et al., 2016. Channel Allocation Method for Multi-radio Wireless Mesh Networks based on a Genetic Algorithm. Hong Kong, International Multi Conference of Engineers and Computer Scientists.

viii. Neema Abraham et al, 2014. Adaptive Channel Allocation Algorithm for Wi-Fi Networks. India, International Conference on Circuit, Power and Computing Technologies.

ix. $\quad$ Public class Wi-FiManager, 2016. Android Developers. [Online] Available at: https://developer.android.com/reference/android/net/Wi-

Fi/WiFiManager.html\#calculateSignalLevel\%28int,\%20int\%29 [Accessed 27 August 2016].

x. Raniwala et al, 2004. Centralized Channel Assignment and Routing Algorithms for MultiChannel Wireless Mesh Networks. ACM SIGMOBILE Mobile Computing and Communications Review, 8(2), pp. 50-65.

xi. $\quad$ Roh, H. T. \& Lee, J. W., 2014. oint Channel and Flow Assignment Algorithm for Wireless Mesh Networks. s.l., Networks, International Conference on Information Networking.

xii. Saleem Iqbal et al, 2015. Channel Allocation in Multi-radio Multi-channel Wireless Mesh Networks: A categorized Survey. KSII Transactions on Internet and Information Systems, 9(6), pp. 1642-1661.

xiii. Shugo Kajita et al, 2014. A Channel Selection Strategy for WLAN in Urban Areas by Regression Analysis. s.l., oth IEEE International Conference on Wireless and Mobile Computing, Networking and Communications .

xiv. Speedtest, 2016. Speed Test. [Online] Available at: http.speedtest.net

xv. Tajuddin, S. A., 2017. Least Congested Channel Recommendation for Uncoordinated Access Point. Korea, International Conference on Advanced Communications Technology.

xvi. Wireshark, 2016. Wireshark. [Online] Available at: https://www.wireshark.org

xvii. WLAN_Available Network Structure, 2016. Microsoft Developer Resources. [Online] Available at: https://msdn.microsoft.com/enus/library/windows/desktop/ms707403\%28v=vs.85\%29.aspx 